\title{
UPDATE
}

\section{SYSTEMATIC REVIEW OF SOCIETAL AND HEALTH SYSTEM COST OF DENGUE IN LATIN AMERICA}

\author{
Mírian Perpétua Palha Dias Parente ${ }^{1}$, Noêmia Teixeira de Siqueira Filha², \\ Fanny Cortes ${ }^{3}$, Alexander Itria $\mathrm{Jr}^{4}$, João Bosco Siqueira $\mathrm{Jr}^{4}$ and Celina Maria \\ Turchi Martelli ${ }^{4,5}$
}

\section{ABSTRACT}

Aim: to conduct a systematic literature review on dengue costs in Latin America, comparing study methodologies, disease costs and the economic impact of dengue in different countries. Methods: the literature search was carried out in the following electronic databases: MEDLINE/ PubMed, EMBASE and LILACS, for the period between 2004 and 2014. To make comparisons possible, the costs identified in the selected studies were converted to local currency values, adjusted to the consumer price index (2014) and converted to purchasing power parity (PPP). Results: 728 publications were identified in databases and 13 papers were selected for analysis. Nine of the thirteen studies were conducted from a societal perspective and three from a health system perspective. In most studies, indirect costs accounted for the largest percentage of total outpatient costs. In contrast, for hospitalized patients, direct medical costs showed the highest percentages. The economic impact of dengue was estimated at I\$ 3.2 billion per year, ranging from I\$ 1.4 to I\$ 5.9 billion, when including the six sub-regions of the Americas. Conclusion: dengue represents a high cost for Latin American society and health system. Studies varied in terms of cost methodology (cost items included, such as direct medical and non-medical and indirect costs, and cost analysis) and the different epidemiological periods in which research was carried out (endemic and/or epidemic).

KEY WORDS: Dengue; costs; health economics; Latin America; review.

\footnotetext{
1. Health Sciences Center, State University of Piaui, Teresina, Piaui, Brazil.

2. Department of Global Health and Development, London School of Hygiene and Tropical Medicine, London, United Kingdom.

3. University of Pernambuco, Recife, Brazil.

4. Oswaldo Cruz Foundation, Aggeu Magalhães Research Center, Recife, Brazil.

5. Department of Public Health, Institute of Tropical Pathology and Public Health, Federal University of Goias, Brazil.

Corresponding author: Mírian Perpétua Palha Dias Parente. Rua Prof. Julieta Neiva Nunes, 5821, Bairro Uruguai, CEP 64073-500, Teresina. Piauí, Brazil. Email: parentepalha@hotmail.com
} 


\section{INTRODUCTION}

Several Latin American countries are currently facing an epidemic of three important arboviruses: dengue, Zika and chikungunya. Besides the devastating effect on the health of the population, the risk of chronicity, early deaths and congenital malformations, these diseases also bring about a major economic impact on the health system and society in general. (CDC, 2016; IVAC, 2016; Paixão et al., 2016). According to the World Health Organization (WHO), 50 to 100 million people are infected with dengue virus every year (WHO, 2009). However, new global estimates go as high as 390 million infections annually (Bhatt et al., 2013). A recent study on the distribution and impact of dengue indicated that American countries account for 40.5 million people infected and 13.3 million cases of dengue fever (Bhatt et al., 2013).

Dengue is considered one of the main public health challenges in the Americas due to the burden of the disease, the difficulty in controlling the vector and its epidemic potential (WHO, 2009; Bhatt et al., 2013; IVAC, 2016). Thus, the need for developing a vaccine to prevent dengue and the recent Zika, dengue and chikungunya epidemics in Latin America, highlights the importance of studying the economic impact of these diseases. Studies addressing cost of illness evaluate the impact of a disease and its comorbidities on the health outcomes and its effect on longevity, morbidity, decrease in health status and quality of life. Furthermore, these studies analyse financial aspects of the disease including direct and indirect expenditures due to premature death or disability. Therefore, disease cost estimates are highly relevant in regard to better allocation of resources and to support economic evaluations, such as cost effectiveness of new technologies for coping with the epidemics, and budget impact analyses used for decision making (Jo, 2014).

Drummond et al. (2005) points out that the cost and burden of disease on society, as well as the years of life lost, are key components in economic evaluations (Drummond et al., 2005; Vanni et al., 2009). However, methodological variability in the different studies could make it difficult to compare results between countries, regions and/or different epidemic periods (Suaya et al., 2009). Recently, the International Vaccine Access Center (IVAC) published a methodological guide for dengue costing studies in the Americas entitled Costing Dengue Cases And Outbreaks: A Guide To Current Practices and Procedures (Armien et al., 2012). These recommendations are the result of a joint effort of health researchers and healthcare managers in endemic countries to make economic studies on dengue comparable (Armien et al., 2012). A systematic review assessing the cost of dengue in dengueendemic countries worldwide (1980-2013) reported substantial costs due to hospitalization and lost earnings and suggested further research in this area (Constenla et al., 2015). 
We conducted systematic literature review of dengue cost studies in Latin America, assessing studies in accordance with published methodological guidelines (Armien et al., 2012) and comparing the economic impact of the disease in different countries. Our review focused on dengue costs in Latin America countries, calculating comparable data from 2004 to 2014.

\section{METHODS}

\section{Literature Review, Data Selection and Extraction}

A literature review of published papers on dengue costs was carried out in the following electronic databases: (1) Medical Literature Analysis and Retrieved System - MEDLINE/PubMED (http://www.ncbi.nlm.nih.gov/ pubmed/); (2) EMBASE (http://www.embase.com/login) and (3) the Latin American and Caribbean Center on Health Sciences Information (LILACS) (http://lilacs.bvsalud.org/), as well as manual bibliographic searches from relevant review papers.

Literature research was conducted by means of descriptors recorded on the Health Sciences Descriptors (DeCS) and the Medical Subject Headings (MeSH) databases. Searches were conducted for terms present on study titles and abstracts in Portuguese, Spanish and English. Boolean operators "AND" and "OR" and quotation marks were used to facilitate the search for documents. The combination of terms used in the search were: "dengue"; "dengue virus"; "severe dengue"; "hemorrhagic dengue"; "dengue hemorrhagic fever"; "hemorrhagic fever dengue"; "dengue shock syndrome"; "classic dengue fever"; "classical dengue"; "cost"; "cost analysis"; "direct costs"; "indirect costs"; "cost measure"; "cost of illness"; "health care costs"; "health expenditure"; "burden of illness"; "cost of disease" and "cost of sickness". Figure shows the complete strategy used in searching for papers. We included original research papers published in international or national journals. We focused on studies conducted in Latin America, published between January $1^{\text {st }}, 2004$ and October $8^{\text {th }}, 2014$. Editorials, conferences and case reports were excluded. Bibliographic references on selected papers were also reviewed to search for documents not displayed in the database search.

Two independent reviewers who read titles and summaries initially selected papers. In case of divergences, a third reviewer decided whether the article would be included. Two independent reviewers (MPPDP and NTSF) for data extraction and subsequent revision by reviewers (CMTM and FC) also read the selected papers in full. The whole group evaluated disagreements in order to reach a common ground on data collection. 


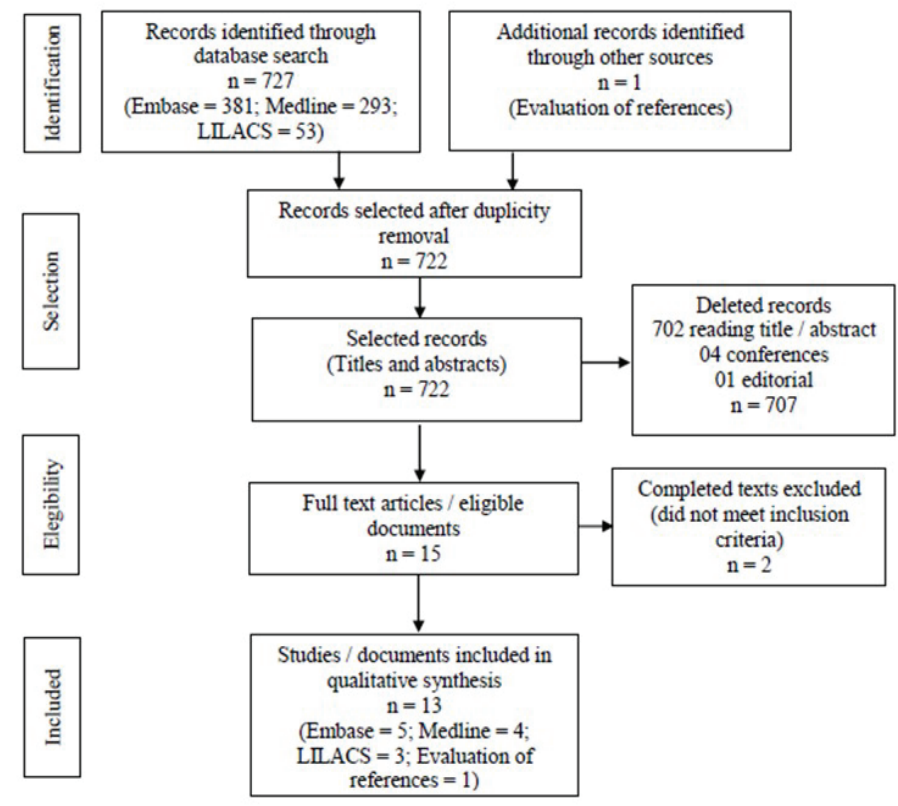

Figure. Results of the bibliographic search in the identification of dengue cost studies in Latin America

An instrument of data collection was developed for extracting data. This collection tool was based on the guide Costing Dengue Cases And Outbreaks: A Guide To Current Practices And Procedures (Armien et al., 2012). The choice of this guide as a model for the data collection sheet was due to the quality of its guidelines regarding dengue-costing studies, despite the fact that it was not designed specifically for systematic review purposes. The variables used were: first author (reference), year of publication, location (city, state and country), sample size, ratio of outpatient: hospital cases, study design (cost analysis, cost-of-illness, cost of outbreak), type of data (primary data, secondary data), study perspective (societal, health system), study period, expansion factor (outpatient and hospital), sensitivity analysis, cost component (direct medical and non-medical costs, indirect cost) for outpatient and inpatient. The expansion factors were those provided by the studies. We also extracted: case definition, sector (public or private), estimated regional and/or national cost, impact on country's GDP, study limitations, and inclusion of vector control program costs and social mobilization (data not shown). 


\section{Data Analysis}

Each cost component was converted to local currency values for each country for the investigated year. Subsequently, the resulting monetary values were adjusted in accordance with the annual consumer price index for all urban healthcare users in 2014. This was done to account for the effects of inflation in that period. (US Department of Labor. US labor statistics data. Consumer Price Index. http://www.bls.gov/cpi/). Next, values were converted according to purchasing power parity (PPP), which is the exchange rate equivalent to the price of a basket of identical goods and services in different countries. Thus, direct inpatient and outpatient health system and societal costs were expressed in international dollars (I\$), which is an hypothetical unit of currency used in order to enable comparison between countries using the US dollar as a common reference. (Organization for Economic Co-operation and Development. Purchasing power parities - PPP -http://www.oecd.org/std/ ppp). Cost conversions per episode of dengue were carried out for studies with primary data (OECD, 2014). Publications that have updated results already published were not converted to avoid duplicate information.

Direct medical (medications, tests) and non-medical (transportation, food) costs were analysed, as well as indirect costs (productivity loss and school days lost) and total cost (sum of indirect and direct costs). The result was expressed in cost per case of dengue fever, which is classified as outpatient and hospitalized cost.

\section{Quality Assessment and Protocol Revision}

The quality of selected papers was assessed according to methodological recommendations described on the Costing Dengue Cases And Outbreaks: $A$ Guide To Current Practices And Procedures (Armien et al., 2012). The quality assessment was focused on the methods and analysed seven criteria described in the guideline. (1) Perspective of analysis: patient/family, provider or societal perspective. The guideline recommends the adoption of the broader societal perspective, with the inclusion of all types of health care providers. (2) Time frame: epidemic and non-epidemic period. The recommendation is to perform the costing study covering both epidemic and non-epidemic periods, as a short period of analysis would not capture variation in costs due to inter-epidemic intervals and seasonal fluctuations. (3) Definition and classification criteria of cases: dengue with and without warning signs and severe dengue. This item is important because it allows the comparison of costs among different types and degrees of severity of dengue disease. (4) Sample size and sample rate calculation: method description and sample representativeness in terms of the study population. This allows the assessment of the representativeness of costs ensuring that the empirical estimate is close to the true value in the 
population under analysis. (5) Cost category: (hospitalized or outpatient), with the guideline recommending the adoption of both categories (6). Classification of costs: direct medical and non-medical costs, indirect costs. The guideline recommends the inclusion of all costs involved in the diagnosis and treatment of dengue in order to have a better understanding of the main contributors to the total cost and its dynamic. (7) Data sources: primary data collection prospective and retrospective, interviews; secondary data collection - published literature, national data. The systematic review protocol was not registered in any systematic review database.

\section{RESULTS}

\section{Selection and Paper Characteristics}

In total, 728 publications regarding the costs of dengue were found, of which 13 papers met the inclusion criteria for the study (Figure 1). Out of all papers included, five came from the EMBASE database; four from MedLine (PubMed); three from LILACS and one from the manual bibliographic search. Of the 20 countries in Latin America, only half of them have measured the economic impact of dengue. Eleven of thirteen publications refer to studies conducted in the following countries: Argentina (1 paper), Brazil (2 papers), Colombia (1), Cuba (2), Nicaragua (1) Panama (1), Puerto Rico (1) and Venezuela (2). Suaya et al. (2009) and Shepard et al. (2011) conducted multicenter studies including several countries in the Americas (Brazil, El Salvador, Guatemala, Mexico, Panama and Venezuela). Publications by Armien et al. (2008) and Suaya et al. (2009) use primary data from one same study to estimate dengue costs in Panama.

Table 1 shows the main characteristics of selected studies. Nine of the thirteen studies were conducted from a societal perspective and three from the health system perspective (public and/or private). The expansion factor (EF) for outpatient and hospitalized cases was used in five studies, but there is large variation between the EF used. This measure is used to adjust the number of dengue cases reported by official health information systems in order to get a more realistic estimate (Undurraga et al., 2013). The highest EF values were recorded for studies in Nicaragua (Wettstein et al., 2012), which assigned an $\mathrm{EF}$ value of 20 for the endemic period and 10 for the non-epidemic period. The multicenter study (Suaya et al., 2009) conducted sensitivity analyses with EF variation $(2,3$ and 6$)$.

Table 2 shows direct medical and non-medical costs and indirect costs

per patient converted to international dollars (2014), divided into outpatient and hospitalized. The cost per dengue case varied greatly in Latin America studies. Two studies of dengue hospitalization costs were performed in Venezuela. Both assessed direct medical costs and one of them also evaluated indirect costs (Añez et al., 2006; Natera et al., 2009). The first study showed that the 
direct medical cost of hospitalized dengue patients was I\$ 137, and indirect costs ranged from I\$ 402 to I\$ 525 for the years 2001 and 2003, respectively. In the second study, direct medical cost estimates for the capital Caracas ranged from I $\$ 1,244$ to I 3,066 for the years 2000 and 2008, respectively (Natera et al., 2009).

In Argentina, the study by Tarragona et al.(2012) evaluated dengue hospital costs in three scenarios during the 2009 outbreak, with lower-cost (first scenario) and higher-cost (third scenario) medical alternatives for patients treated in intensive care units (ICU) in different regions of the country. Direct medical costs ranged from I\$ 242 to I\$ 872 (ICU) and indirect costs ranged from I $\$ 277$ to I $\$ 320$ for labour loss and from I\$ 24 to I\$ 35 for school days lost, depending on the region (Tarragona et al., 2012).

In Guantanamo, Cuba, dengue hospital cost was estimated at I\$ 1,213. The direct medical cost was I $\$ 752$, which represents $\sim 62 \%$ of the total, followed by the indirect cost: I\$ $352(\sim 29 \%)$ (Baly et al., 2012). A study undertaken in two cities of Colombia estimated that severe dengue cases had costs of more than $60 \%$ compared to less severe hospitalized cases (I\$ 2,031 versus I\$ 1,283) (Castañeda-Orjuela et al., 2012). Valdés et al. (2012) carried out a partial economic evaluation for hospitalized dengue cases during an epidemic period in Santiago de Cuba, where the total costs for hospitalization showed $79.8 \%$ of direct medical costs (Valdés et al., 2012).

In Puerto Rico, Halasa et al. (2012) evaluated the outpatient and hospital costs of dengue fever. The cost of a dengue episode for outpatients was I\$ 1,944 , of which approximately $70 \%$ accounted for indirect costs and $30 \%$ for direct medical and non-medical costs. For hospitalized patients, about $70 \%$ of total costs were direct medical costs. Hospital costs were about four times higher than outpatient costs (Halasa et al., 2012). In Nicaragua, the total cost per patient, both outpatient and hospital, ranged from I\$ 373 to I\$ 797 (Wettstein et al., 2012).

Machado et al. (2014) conducted a study in a public hospital and three private hospitals in the city of Dourados, Mato Grosso do Sul (MS), in the Midwest region of Brazil. This work estimated direct medical costs at I\$ 554 (Machado et al., 2014). The study by Pereira et al. (2014) evaluates dengue episode costs after the 2011 floods in the city of Nova Friburgo, state of Rio de Janeiro. Direct medical costs were estimated at I\$ 342 and indirect costs ranged between I\$ 97 and I\$ 166, according to the national minimum wage (2011) and the city's average income, respectively. In this study, the authors show an increase of at least 30 times in the number of reported dengue cases after this environmental disaster, compared to previous years (Pereira et al., 2014). 
In the multicenter study by Suaya, Castaneda-Orjuela and Halasa, the total hospital cost was higher than outpatient cost. Hospital costs varied from I\$ 8,355 to I\$ 917 for Puerto Rico and Guatemala, respectively. The direct medical costs component accounted for approximately $70 \%$ of the total cost, except for Brazil $(\sim 50 \%)$. The total cost for outpatients showed inter-country variation from I\$ 1,944 to I\$ 193 for Puerto Rico and Guatemala, respectively. The conversion table of dengue cost components to international dollars did not include publications by Armien (Panama) and Shepard (multicenter), whose results were in the publication by Suaya et al. (2009).

Graph summarizes cost components of a dengue episode for outpatients and hospitalized patients. Most studies reported outpatient costs. Hospitalization cost was the biggest cost component in the cost of dengue treatment in all selected studies. For ambulatory cases, indirect cost was the main component of total costs. In contrast, for hospitalized patients, direct medical cost was the main component of the total costs.

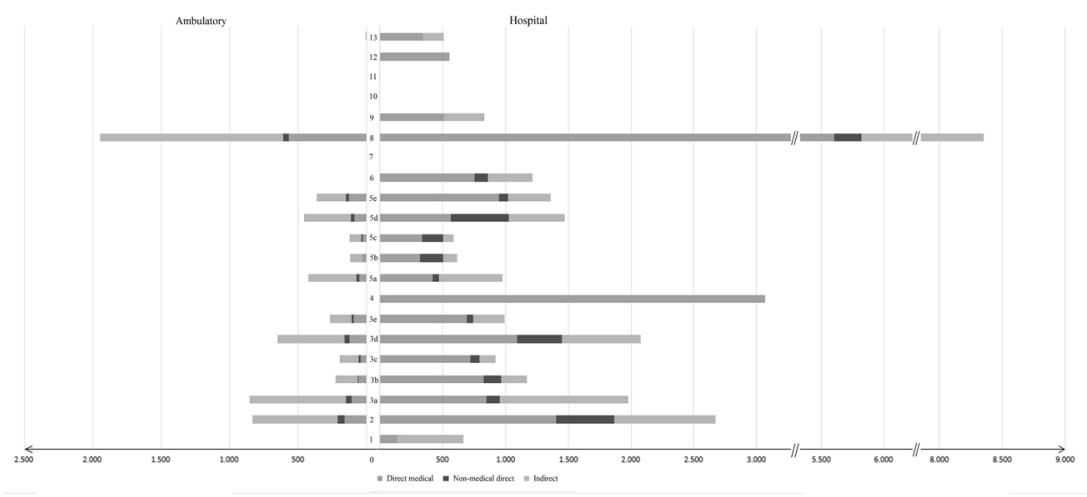

Graph. Cost of dengue (International dollars, I\$) by ambulatory and hospital according to the studies selected, Latin America (2004-2014). 


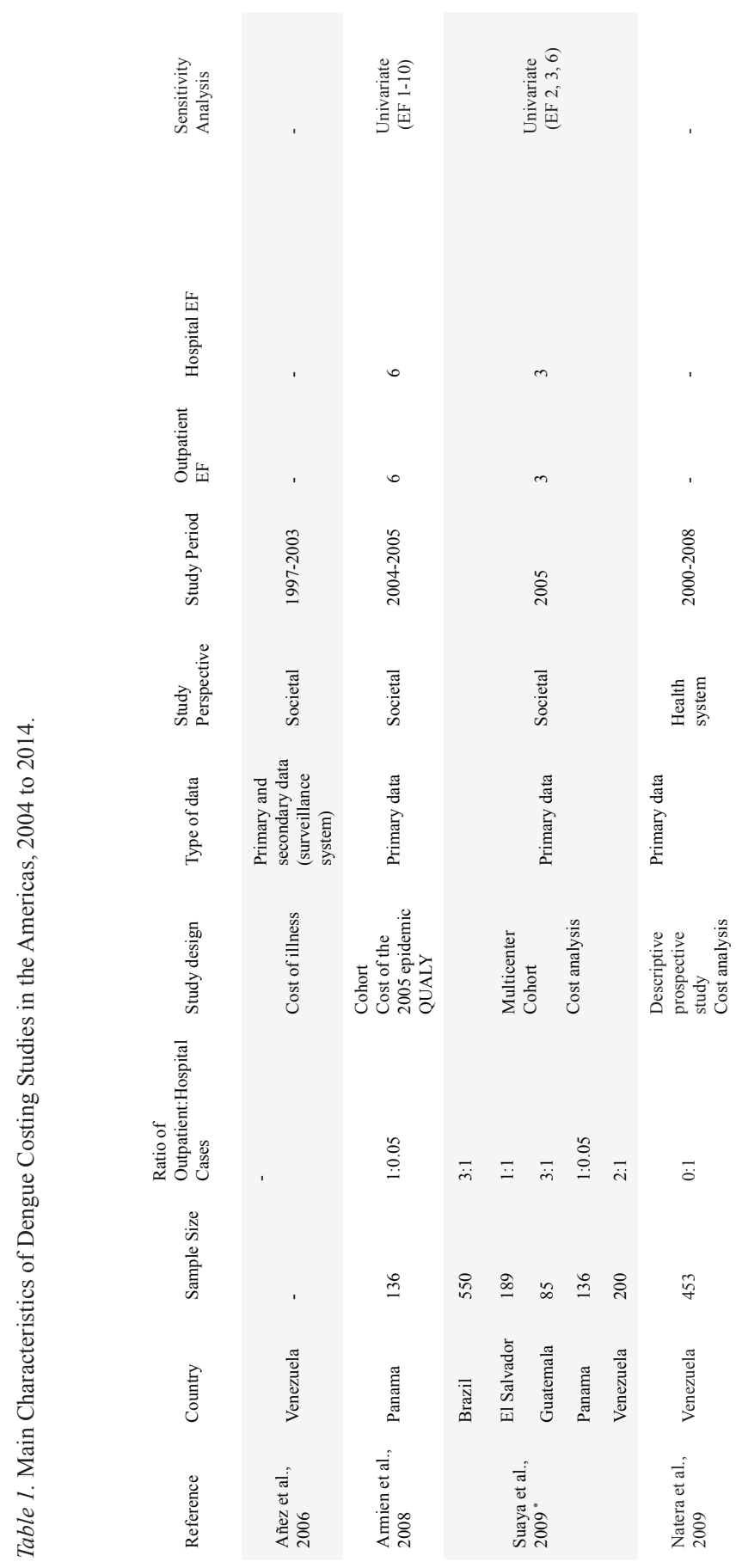




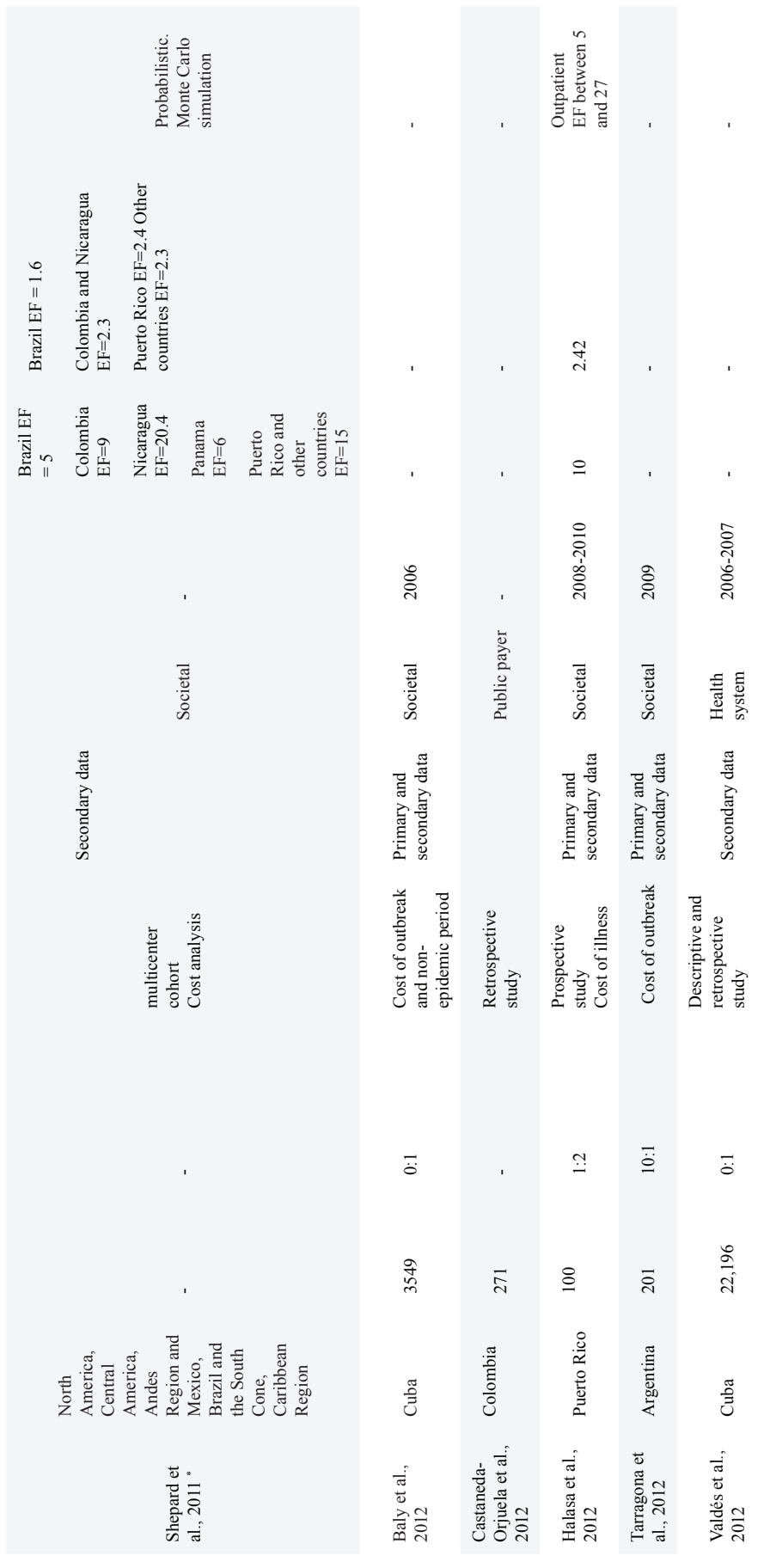




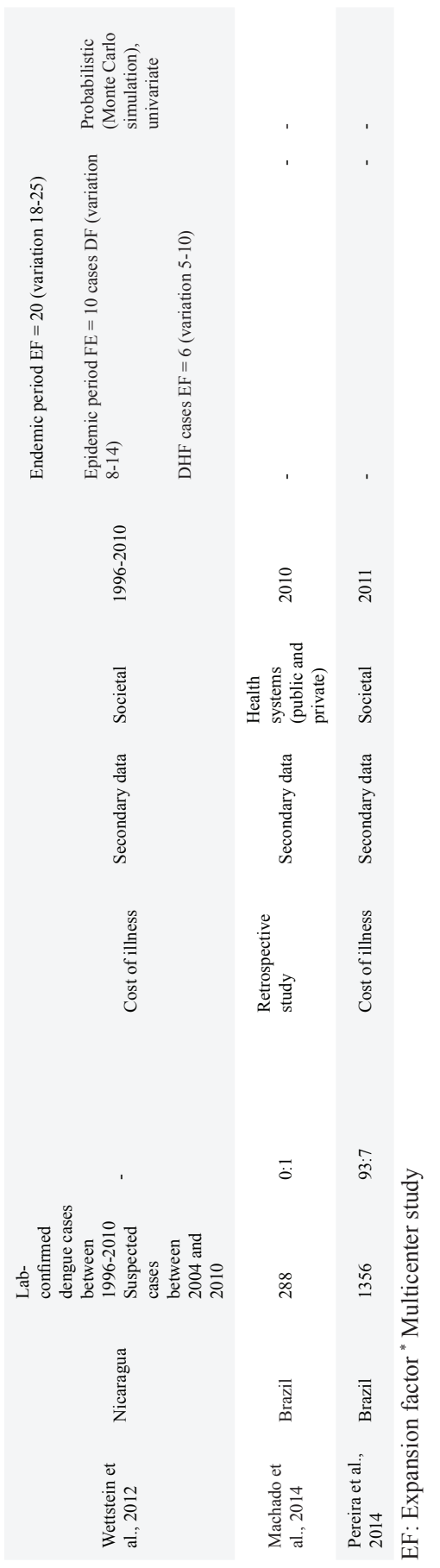




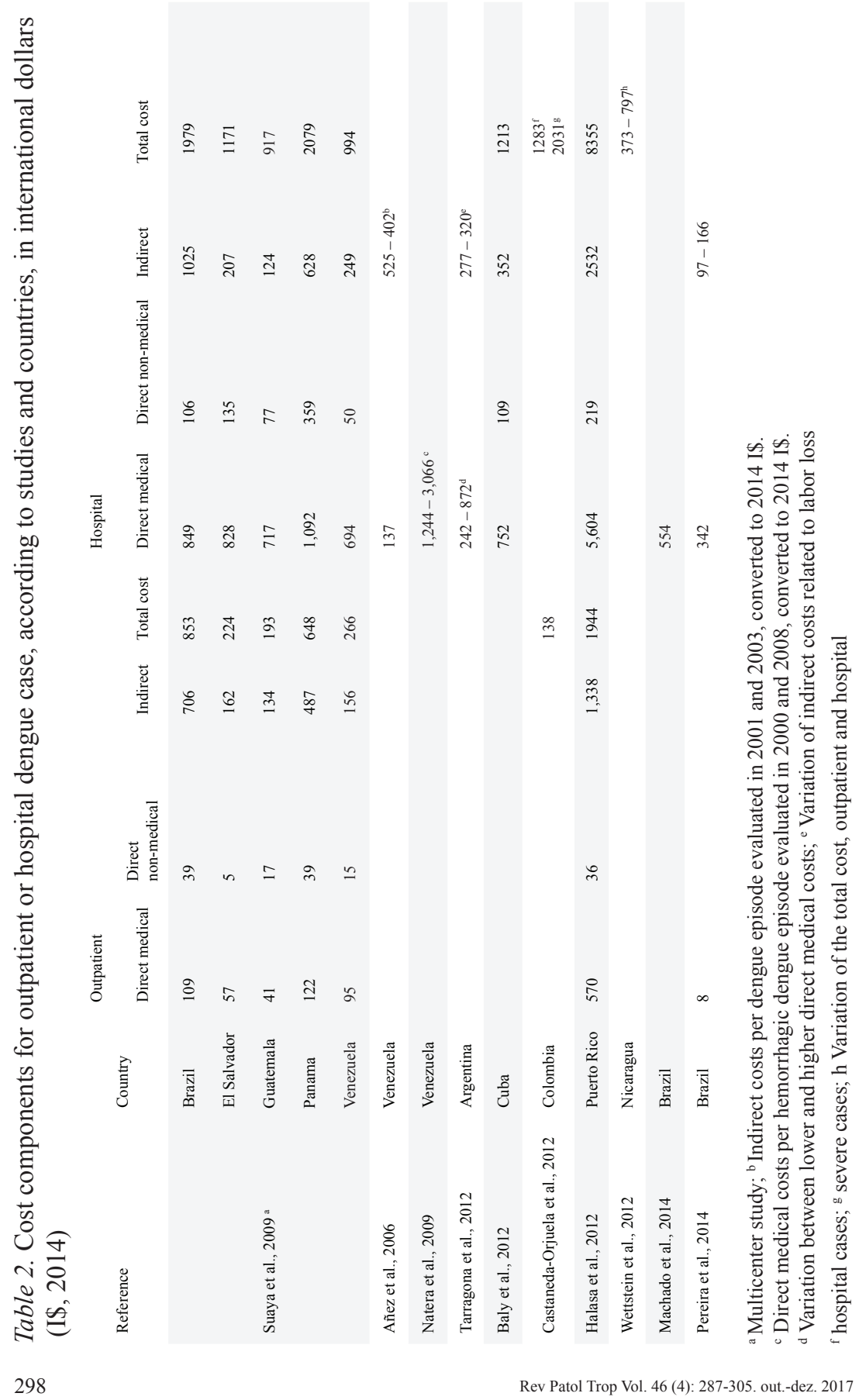




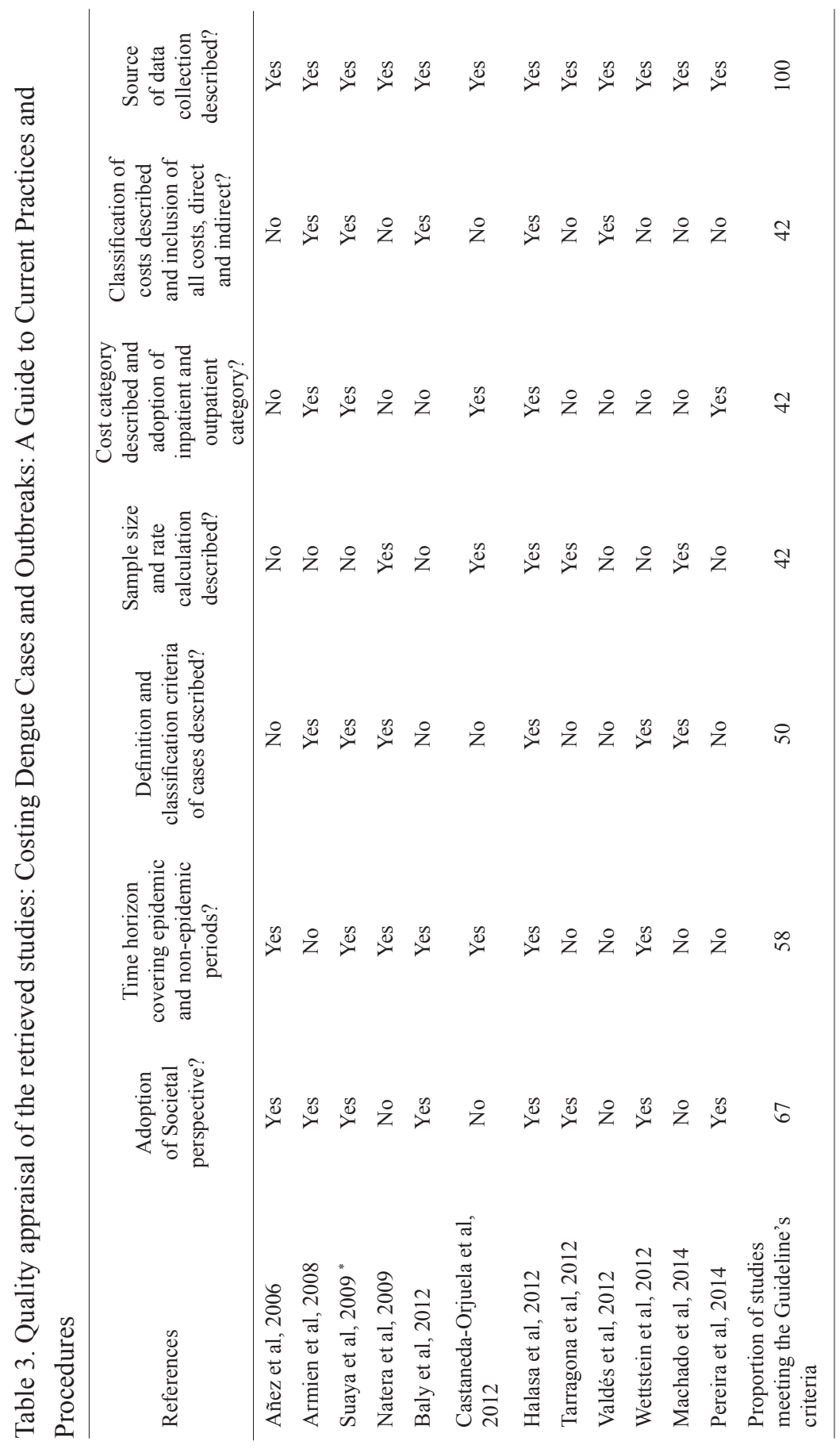




\section{Quality Assessment}

Only the study by Shepard et al. (2011) did not carry out a quality assessment because it was a systematic literature review. For this type of study, other quality assessment methods must be applied. The quality assessment identified that most studies were carried out from a societal perspective $67 \%$ (8/12). Four studies considered provider perspective. Most of them 58\% (7/12) also covered both the epidemic and non-epidemic periods and all of them reported data collection sources adequately. Other quality aspects were also assessed and the conclusion was that most studies did not follow the criteria established by the reference guide. These quality aspects were the following: a) criteria for defining and classifying reported cases (6/12); b) sample size calculation with method description and representativeness of sample according to study population (5/12); c) description of outpatient and hospital cost categories (5/12) and; d) description of cost classification (direct medical and non-medical costs and indirect costs) (5/12). Table 3 gives a detailed description of the quality assessment.

\section{DISCUSSION}

In the Americas the first multicenter study from a societal perspective was undertaken simultaneously in five countries (Brazil, Venezuela, El Salvador, Guatemala, Panama) in 2005 (Suaya et al., 2009). The total cost per outpatient and hospital episode showed inter-country variation. Indirect costs accounted for the largest percentage of the total outpatient cost. For hospitalized dengue patients, the greatest part of the total costs was direct medical costs. Considering that the study used a common research protocol, variability in cost per episode of dengue appears to reflect differences in local healthcare provision, type of healthcare access and economic conditions of each country. The authors estimated a total of I $\$ 343$ million in aggregate morbidity and mortality costs due to dengue (2001-2005) in these five American countries. In another study (Shepard et al., 2011) the economic impact of dengue was estimated at I\$ 3.2 billion per year, ranging from I\$ 1.4 to I\$ 5.9 billion, when including six sub-regions of the Americas: North America (United States and Canada); Central America and Mexico; Andes region (Bolivia, Colombia, Ecuador, Peru, and Venezuela); Brazil and the South Cone (Argentina, Chile, Paraguay and Uruguay); and the Caribbean. This update of the economic impact of dengue used cost results from the multicenter study (Suaya et al., 2009) updating values for 2010 and transferring them to other countries in the region.

The multicenter study conducted in different countries of the Americas made it possible to compare direct and indirect costs of a disease episode in different regional epidemiological contexts (Suaya et al., 2009). In this study, 
the average cost per hospitalized case was estimated at approximately I\$ 1,700 in Panama and Brazil versus I\$ 750 in Guatemala. Intra-country comparisons showed that the average cost per hospitalized case was about three times higher than the cost of an outpatient case. In outpatient cases, indirect costs caused by productivity loss for patients and families and school days lost were higher than the direct medical costs. In this study, Brazil was represented by a capital city in the Midwest region and the research was carried out in 2005, with circulation of DENV-1, DENV-2 and DENV-3, before the rise of dengue transmission in the region.

After the period addressed by this review (2014), recent publications on the impact of dengue in Colombia (Rodriguez et al., 2015), México (Undurraga et al., 2015) and Brazil (Martelli et al., 2015) should also be discussed. The cost of dengue in Colombia was estimated at approximately US\$ 168 million in the 2010 epidemic (Rodriguez et al., 2015). In this study, direct medical costs of a dengue episode accounted for most of the total costs of hospitalized patients while indirect costs associated with patient or caregiver production loss accounted for the largest share of costs per outpatient case. This proportional distribution of cost components was similar to that found in previous studies in other countries of the Americas (Shepard et al., 2011; Suaya et al., 2009).

Recently, a study of dengue burden in Mexico evaluated disease and prevention costs using multiple data sources (Undurraga et al., 2015). In addition to the DALY burden of disease, a sensitivity analysis of other parameters was also undertaken (expansion factor, unit costs, days of work lost, use of health services, among others) to assess possible uncertainties of economic estimates. Estimates broken down by cases and deaths were presented for the period between 1995 and 2011. In Mexico, the average annual cost of dengue was estimated at US\$ 170 million.

In Brazil, the overall cost of dengue from a societal perspective reached US\$ 1.2 billion in the 2012-2013 epidemic (Martelli et al., 2015). These high values include medical costs, productivity loss and deaths considering adjustments of reported cases. We believe that this national multicenter study was the most representative dengue impact evaluation from a societal perspective using data from interviews of about two thousand suspected cases of dengue, both outpatients and/or hospitalized, in the public and private sector, and covering the four most endemic areas in the country (North; Northeast, Midwest and Southeast). The cost of a dengue episode showed inter-region variation depending on the type of health services provided, the incidence and/ or severity of the disease, the proportion of public and private sector healthcare provision and the population's socioeconomic status. In this study, $67 \%$ of the total costs were related to outpatient costs. Thus, dengue had a significant economic impact, especially due to the great demand for outpatient care and disease-related productivity loss. Although the average cost of a hospitalized dengue case was greater than the outpatient cost. Only $10 \%$ of all dengue 
cases were hospitalized in the studied period (Martelli et al., 2015). Overall, productivity loss has been identified as one of the main cost components of dengue measured as an indirect cost component, which reinforces the need to assess the impact of dengue from a societal perspective (Constenla et al., 2015; Undurraga et al., 2015; WHO, 2009).

It is worth noting the variability in dengue episode costs among different American countries as well as within the same country. This is true even for multicenter studies using the same research protocol (Suaya et al., 2009). Different inclusion criteria for cases (suspected and/or confirmed by lab test) can affect the disease cost, which makes it difficult to compare the impact of the disease.

Estimates of the overall costs of dengue depended on records of dengue cases reported by surveillance systems (Shepard et al., 2011). Epidemiological aspects, such as disease seasonality (endemic and epidemic periods) and characteristics of surveillance systems should be taken into account when interpreting the overall cost estimates (Runge-Ranzinger et al., 2014; Stahl et al., 2013). For example, in Brazil, dengue is characterized by a growing time series trend with a wide geographical dispersion between 2000 and 2010 (Teixeira et al., 2013). About one million cases of dengue were reported in 2010, while two million cases were recorded in the epidemic of the year 2013 (Brasil, 2013).

For dengue economic impact studies, it is recommended to include epidemic and endemic periods and a wide time frame (years) in order to compare different periods (WHO, 2009). Three studies included in our systematic review included cost of illness for outbreak (Armien, Baly, Tarragona). This strategy has been applied in several costing studies that include a historic series of cases reported for national cost assessments (Martelli et al., 2015; Undurraga et al., 2015). In order to adjust the data regarding notifications of the passive surveillance system, an expansion factor is used, which is the ratio between existing symptomatic cases of dengue fever and the number of cases registered by the notification system. In general, the EF for outpatient cases is greater than the $\mathrm{EF}$ for hospitalized cases. In addition, there are variations between endemic and epidemic periods. A recent review of EFs that quantify underreporting of dengue in surveillance systems of different endemic countries showed an information gap in Brazil (Toan et al., 2015). Our systematic review identified six studies that used EF to adjust for underreporting an important issue in many dengue-endemic countries. Different expansion factors are chosen for outpatient and inpatient, sometimes based on literature or expert opinion. The huge variation between $\mathrm{EF}$ shows there is little agreement between experts on the value to be used to adjust for missing cases.

In conclusion, our systematic review showed that dengue represents a high cost for Latin American health systems and society. The costs of dengue episodes varied among different studies, due to the characteristics of 
surveillance systems in each country, and to whether studies were carried out in endemic or epidemic periods. Methodological variability makes it difficult to compare studies between countries/regions and/or in different epidemic periods. Differences were observed across economic studies: design, data sources, cost components and outcomes measured and dengue case definition. Few costs studies included surveillance costs (Tarragona, Baly). As mentioned by Constenla et al (2015) "there is a need for standardized guidelines describing appropriate sources, minimum cost components and use of proxy cost estimates when data are not available from the country or region under analysis". In general, school absenteeism and productivity loss represented a significant portion of dengue episode costs. Moreover, if one considers the dynamic characteristics of dengue epidemiology in the Americas, other economic studies regarding this disease are also recommended.

\section{REFERENCES}

1. Añez G, Balza R, Valero N, Larreal Y. Impacto económico del dengue y del dengue hemorrágico en el Estado de Zulia, Venezuela, 1997-2003. Rev Panam Salud Pública 19: 314-320, 2006.

2. Armien B, Suaya JA, Quiroz E, Sah BK, Bayard V, Marchena L, Campos C, Shepard DS. Clinical Characteristics and National Economic Cost of the 2005 Dengue Epidemic in Panama. Am J Trop Med Hyg 79: 364-371, 2008.

3. Armien B, Arredondo J, Carabali M, Carrasquilla G, Castro R, Durand L, Durán L, García ME, Gallegos V, Gontes M, López JG, McFarlane C, Montoya R, Sartori AMC, Siqueira JB, Martelli C, Constenla D. Costing Dengue Cases And Outbreaks: A Guide To Current Practices And Procedures International Vaccine Access Center (IVAC). 2012. Baltimore: [s.n.]. Available at: <http://www.jhsph.edu/research/centers-and-institutes/ivac/projects/costingdengue-cases-outbreaks-guidelines.pdf>. Access on: 20 Oct. 2014.

4. Baly A, Toledo ME, Rodriguez K, Benitez JR, Rodriguez M, Boelaert M, Vanlerberghe V, Van der Stuyft P. Costs of dengue prevention and incremental cost of dengue outbreak control in Guantanamo, Cuba. Trop Med Int Health 17: 123-132, 2012.

5. Bhatt S, Gething PW, Brady OJ, Messina JP, Farlow AW, Moyes CL, Drake JM, Brownstein JS, Hoen AG, Sankoh O, Myers MF, George DB, Jaenisch T, Wint GR, Simmons CP, Scott TW, Farrar JJ, Hay SI. The global distribution and burden of dengue. Nature 496: 504-507, 2013.

6. Brasil. Ministério da Saúde. Dengue - Informações técnicas. Available at: $<\mathrm{http}: / /$ portalsaude. saude.gov.br/index.php/informacoes-tecnicas-dengue>. Access on: 6 June 2016.

7. Brasil. Ministério da Saúde. Secretaria de Vigilância em Saúde. Departamento de Analise de Situação em saúde. Saúde Brasil 2012: Uma analise de situação de saúde e dos 40 anos do Programa Nacional de Imunizações. Capitulo 6 Doenças com Potencial Epidemia no Brasil 2000 a 2009-2013. Brasília: Editora do Ministério da Saúde. 2013. p 189-211.

8. Castañeda-Orjuela C, Díaz H, Alvis-Guzman N, Olarte A, Rodriguez H, Camargo G, De la Hoz-Restrepo F. Burden of Disease and Economic Impact of Dengue and Severe Dengue in Colombia, 2011. Value Health Reg Issues 1: 123-128, 2012.

9. Center for Disease Control and Prevention (CDC). Chikungunya in the Caribbean. Available at: $<$ http://wwwnc.cdc.gov/travel/notices/watch/chikungunya-caribbean.>. Access on: 6 June 2016.

10. Constenla D, Garcia C, Lefcourt N. Assessing the Economics of Dengue: Results from a Systematic Review of the Literature and Expert Survey. Pharmacoeconomics 33: 1107-1135, 2015. 
11. Drummond M, Sculpher MJ, Torrance GW. Methods for the Economic Evaluation of Health Care Programmes. Chapter 2. Basic types of economic evaluation. Oxford University Press, New York, 2005. p 8-26.

12. Halasa YA, Shepard DS, Zeng W. Economic Cost of Dengue in Puerto Rico. Am J Trop Med Hyg 86: 745-752, 2012.

13. IVAC. International Vaccine Acces Center. The Potential Economic Impact of the Zika Virus. Available at: $<$ http://www.jhsph.edu/research/centers-and-institutes/ivac/IVACBlog/thepotential-economic-impact-of-the-zika-virus>. Access on: 20 June 2016.

14. Jo C. Cost-of-illness studies: concepts, scopes, and methods. Clin Mol Hepatol 20: 327-337, 2014.

15. Machado AAV, Estevan AO, Sales A, Brabes KC, Croda J, Negrão FJ. Direct Costs of Dengue Hospitalization in Brazil: Public and Private Health Care Systems and Use of WHO Guidelines. PLoS Neglected Trop Dis 8: e3104, 2014.

16. Martelli CM, Siqueira JB Jr, Parente MP, Zara AL, Oliveira CS, Braga C, Pimenta FG Jr, Cortes F, Lopez JG, Bahia LR, Mendes MC, da Rosa MQ, de Siqueira Filha NT, Constenla D, de Souza WV. Economic Impact of Dengue: Multicenter Study across Four Brazilian Regions. PLoS Negl Trop Dis 9: e0004042, 2015.

17. Natera I, Garrido E, Martín A. Consecuencias del dengue hemorrágico en niños. Bol Venez Infectol 20: 17-23, 2009.

18. OECD. Prices and purchasing power parities (PPP). 2014. Available at: $<$ http://www.oecd. org/std/ppp>. Access on: 14 Oct. 2014.

19. Paixão ES, Paixão ES, Barreto F, Teixeira Mda G, Costa Mda C, Rodrigues LC. History, Epidemiology, and Clinical Manifestations of Zika: A Systematic Review. Am J Public Health 106: 606-612, 2016.

20. Pereira CAR, Barata MM, Hoelz Mde P, Medeiros VN, Marincola Fde C, Costa Neto C, Marinho DP, Oliveira TV, Trigo AG, Medeiros TK. Avaliação econômica dos casos de Dengue atribuídos ao desastre de 2011 em Nova Friburgo (RJ), Brasil. Cien Saúde Colet 19: 36933704, 2014.

21. Rodriguez RC, Galera-Gelvez K, López Yescas JG, Rueda-Gallardo JA. Costs of Dengue to the Health System and Individuals in Colombia from 2010 to 2012. Am J Trop Med Hyg 92: 709-714, 2015.

22. Runge-Ranzinger S, McCall PJ, Kroeger A, Horstick O. Dengue disease surveillance: an updated systematic literature review. Trop Med Int Health 19: 1116-1160, 2014.

23. Shepard DS, Coudeville L, Halasa YA, Zambrano B, Dayan GH. Economic Impact of Dengue Illness in the Americas. Am J Trop Med Hyg 84: 200-207, 2011.

24. Stahl HC, Butenschoen VM, Tran HT, Gozzer E, Skewes R, Mahendradhata Y, RungeRanzinger S, Kroeger A, Farlow A. Cost of dengue outbreaks: literature review and country case studies. BMC Public Health 13: 1048, 2013.

25. Suaya JA, Shepard DS, Siqueira JB, Martelli CT, Lum LC, Tan LH, Kongsin S, Jiamton S, Garrido F, Montoya R, Armien B, Huy R, Castillo L, Caram M, Sah BK, Sughayyar R, Tyo KR, Halstead SB. Cost of dengue cases in eight countries in the Americas and Asia: a prospective study. Am J Trop Med Hyg 80: 846-855, 2009.

26. Tarragona S, Monteverde M, Marchioni S, Caporale J, Pereiro AC, Palacios JM. Dengue en la Argentina: un análisis económico del impacto de la epidemia de 2009. Salud Colectiva 8: 151-162, 2012.

27. Teixeira MG, Siqueira JB Jr, Ferreira GL, Bricks L, Joint G. Epidemiological Trends of Dengue Disease in Brazil (2000-2010): A Systematic Literature Search and Analysis. PLoS Neglected Trop Dis 7: e2520, 2013. 
28. Toan NT, Rossi S, Prisco G, Nante N, Viviani S. Dengue epidemiology in selected endemic countries: factors influencing expansion factors as estimates of underreporting. Trop Med Int Health 20: 840-863, 2015.

29. Undurraga EA, Betancourt-Cravioto M, Ramos-Castañeda J, Martínez-Vega R, MéndezGalván J, Gubler DJ, Guzmán MG, Halstead SB, Harris E, Kuri-Morales P, Tapia-Conyer R, Shepard DS. Economic and Disease Burden of Dengue in Mexico. PLOS Neglected Trop Dis 9: e0003547, 2015.

30. Undurraga EA, Halasa YA, Shepard DS. Use of Expansion Factors to Estimate the Burden of Dengue in Southeast Asia: A Systematic Analysis. PLoS Neglected Trop Dis 7: e2056, 2013.

31. Valdes AR, Diaz YA, Sanchez DG. Economic evaluation of patient care in the epidemic of dengue. Available at: $<\mathrm{http} / / /$ www.medigraphic.com/pdfs/medisan/mds-2012/mds $125 \mathrm{c} . \mathrm{pdf}>$. Access on: 27 July 2016.

32. Vanni T, Luz PM, Ribeiro RA, Novaes HM, Polanczyk CA. Avaliação econômica em saúde: aplicações em doenças infecciosas. Cad Saúde Pública 25: 2543-2552, 2009.

33. Wettstein ZS, Fleming M, Chang AY, Copenhaver DJ, Wateska AR, Bartsch SM, Lee BY, Kulkarni RP. Total economic cost and burden of dengue in Nicaragua: 1996-2010. Am J Trop Med Hyg 87: 616-622, 2012.

34. WHO. Dengue: Guidelines for diagnosis, treatment, prevention and control. Chapter 1. Epidemiology, burden of disease and transmission. New Edition: Geneve. 2009. p 3-17. 